MADPH-04-1367

ILL-(TH)-04-3

NSF-KITP-04-38

hep-ph/0404182

\title{
Scale of Quantum Gravity
}

\author{
Tao $\operatorname{Han}^{1,3,4}$ and Scott Willenbrock ${ }^{2,3}$ \\ ${ }^{1}$ Department of Physics, University of Wisconsin 1150 University Avenue, Madison, WI 53706, USA \\ ${ }^{2}$ Department of Physics, University of Illinois at Urbana-Champaign 1110 West Green Street, Urbana, IL 61801, USA \\ ${ }^{3}$ Kavli Institute for Theoretical Physics, University of California Santa Barbara, CA 93106, USA \\ ${ }^{4}$ Institute of Theoretical Physics, Academia Sinica, Beijing 100080, P. R. China
}

\begin{abstract}
In the effective field theory of quantum gravitation coupled to $N_{s}$ scalars, $N_{f}$ fermions, and $N_{V}$ vectors, tree unitarity is violated at an energy squared of $E_{C M}^{2}=20\left(G_{N} N\right)^{-1}$, where $N \equiv$ $\frac{2}{3} N_{s}+N_{f}+4 N_{V}$ and $G_{N}$ is Newton's constant. This is related to radiative corrections proportional to $G_{N} N E^{2}$ (where $E$ is the typical energy), due to loops of such particles. New physics must enter before $E_{C M} \approx 6 \times 10^{18} \mathrm{GeV}$ in the standard model, and $4 \times 10^{18} \mathrm{GeV}$ in the minimal supersymmetric standard model.
\end{abstract}

Shortly after discovering the fundamental constant of quantum mechanics that now bears his name, Planck noticed that it may be combined with the fundamental constants of relativity and gravitation to make a constant with the units of mass. This mass also bears his name, $M_{P l} \equiv\left(\hbar c / G_{N}\right)^{1 / 2} \approx 10^{19} \mathrm{GeV} / \mathrm{c}^{2}$. While he could not know the physical significance of this extremely large mass, he did remark that it could be considered as the fundamental unit of mass.

A century later, we still do not know the true physical significance of the Planck mass. Instead, we regard the Planck mass in the context of an effective quantum field theory of gravitation 1, 2, 3]. At leading order, this theory is simply Einstein's theory of general relativity. Beyond leading order, there are corrections to the predictions of Einstein's theory proportional to powers of $E^{2} / M_{P l}^{2}$, where $E$ is the typical energy of the process under consideration (here and henceforth we work with units where $\hbar=c=1$, for convenience). Thus the expansion parameter of this effective theory is proportional to $G_{N} E^{2}$. This expansion, and hence the usefulness of the effective theory, breaks down at energies of order the Planck mass.

In this paper we endeavor to make this statement more precise. We use tree unitarity to determine the energy at which the effective theory of quantum gravity breaks down. While dimensional analysis dictates that the theory breaks down at energies of order $M_{P l}$, it does not reveal the presence of dimensionless constants. Does the expansion break down at an energy close to the Planck mass, $M_{P l} \approx 10^{19} \mathrm{GeV}$, the reduced Planck mass, $M_{P l} / \sqrt{8 \pi} \approx 2.4 \times 10^{18}$ $\mathrm{GeV}$ (which appears naturally in Einstein's theory [4]), or perhaps some altogether different energy?

When the effective theory breaks down, a new description of the physics becomes appropriate. This new description may become relevant before the energy at which the effective theory breaks down. Thus the energy at which the effective theory breaks down places an upper bound on the energy at which new physics must enter. Unitarity has been used to determine the energy at which other effective field theories break down, such as the Fermi theory of the weak interaction [5], the spontaneously-broken electroweak theory (with no Higgs boson) []. , and the standard model in higher dimensions []].

The partial-wave amplitude for a $2 \rightarrow 2$ elastic scattering amplitude satisfies the unitarity condition $\left|\operatorname{Re} a_{J}\right| \leq 1 / 2$, where $J$ is the total angular momentum. If the leading-order contribution to the amplitude violates this condition, then the expansion cannot converge and the effective theory is useless. Our approach is thus to calculate all $2 \rightarrow 2$ scattering amplitudes in quantum gravity and to ask at what energy they violate unitarity.

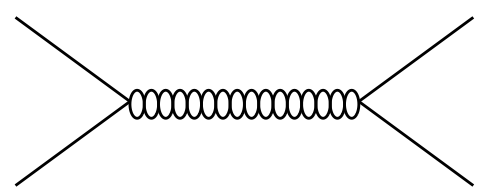

FIG. 1: Scattering of elementary particles via $s$-channel graviton exchange. 
The standard model contains scalar, fermion, and vector fields, with couplings to gravity that depend only on the particles' masses and momenta. At energies of order the Planck mass, the particles' masses are negligible. For convenience, we calculate the scattering amplitudes in the symmetric phase of the electroweak theory, where all particle masses vanish.

We begin with the scattering of scalar particles, $s \bar{s} \rightarrow s^{\prime} \bar{s}^{\prime}$. There are two such (complex) scalar particles in the standard model, corresponding to the two components of the Higgs doublet field. We consider the case $s \neq s^{\prime}$, in which there is only an $s$-channel diagram, as shown in Fig. 1 There are two reasons for doing so. First, amplitudes which include a $t$ - or $u$-channel diagram do not yield a well-defined partial-wave amplitude, since the integration over the scattering angle diverges at $\theta=0$ or $\pi$. This corresponds to an infrared singularity due to the exchange of an onshell graviton, and is an irrelevant complication which we choose to avoid. Second, the fraction of processes in which $s=s^{\prime}$ diminishes as the number of particles increases. There are many fields in the standard model, so neglecting this small subset of processes is a good approximation. It is for these same reasons that we neglect processes of the type $s s^{\prime} \rightarrow s s^{\prime}$.

The scattering of scalar particles is actually the most subtle case, since their coupling to gravitons is not fixed uniquely by their mass and momenta at leading order. The coupling of a scalar field to gravity is given at leading order by

$$
\sqrt{-g} \mathcal{L}=\frac{1}{16 \pi G_{N}} \sqrt{-g}(-2 \lambda+R)+\sqrt{-g}\left(g^{\mu \nu} \partial_{\mu} \phi^{\dagger} \partial_{\nu} \phi-m^{2}|\phi|^{2}+a R|\phi|^{2}\right)+\ldots
$$

where $g$ is the determinant of the metric, $\lambda$ is the cosmological constant, $R$ is the Ricci scalar, $m$ is the scalar mass, and $a$ is a free parameter. We do not know the value of $a$ in the standard model, so we leave it as a free parameter. It is only for a scalar field that such an ambiguity exists. The case $m=0, a=-1 / 12$ corresponds to the conformal limit of the theory [8].

The amplitude for $s$-channel scalar-scalar scattering, at energies much greater than the scalar mass, is

$$
\mathcal{A}\left(s \bar{s} \rightarrow s^{\prime} \bar{s}^{\prime}\right)=-\frac{4}{3} \pi G_{N} E_{C M}^{2}\left[d_{0,0}^{2}-(1+12 a)^{2} d_{0,0}^{0}\right]
$$

where $E_{C M}$ is the total center-of-momentum energy and $d_{\mu, \mu^{\prime}}^{J}$ is the Wigner $d$ function, where $J$ is the total angular momentum, $\mu=\lambda-\bar{\lambda}$ is the difference of the helicities of the initial-state particles, and $\mu^{\prime}=\lambda^{\prime}-\bar{\lambda}^{\prime}$ is the difference of the helicities of the final-state particles. The first term corresponds to $J=2$ exchange, while the second corresponds to $J=0$. The $J=0$ component of a virtual graviton couples to the trace of the energy-momentum tensor, which explains why the $J=0$ term vanishes in the conformal limit $(a=-1 / 12)$, where $T_{\mu}^{\mu}=0$.

The presence of a $J=0$ component of a virtual graviton does not violate any physical principles. While a real graviton must contain only helicity \pm 2 components $[9,10,11$, a virtual graviton can contain a $J=0$ component. There is no violation of angular-momentum selection rules in quantum gravity, as recently claimed in Ref. [12]. This claimed was based on the assumption that a virtual graviton cannot have a $J=0$ component.

\begin{tabular}{|c|c|c|c|c|c|}
\hline & $s^{\prime}{ }^{\prime}$ & $f_{+}^{\prime} \bar{f}_{-}^{\prime}$ & $f_{-}^{\prime} \bar{f}_{+}^{\prime}$ & $V_{+}^{\prime} V_{-}^{\prime}$ & $V_{-}^{\prime} V_{+}^{\prime}$ \\
\hline$s \bar{s} \rightarrow$ & $2 / 3 d_{0,0}^{2}-2 / 3(1+12 a)^{2} d_{0,0}^{0}$ & $\sqrt{2 / 3} d_{0,1}^{2}$ & $\sqrt{2 / 3} d_{0,-1}^{2}$ & $2 \sqrt{2 / 3} d_{0,2}^{2}$ & $2 \sqrt{2 / 3} d_{0,-2}^{2}$ \\
\hline$f_{+} \bar{f}_{-} \rightarrow$ & $\sqrt{2 / 3} d_{1,0}^{2}$ & $d_{1,1}^{2}$ & $d_{1,-1}^{2}$ & $2 d_{1,2}^{2}$ & $2 d_{1,-2}^{2}$ \\
\hline$f_{-} \bar{f}_{+} \rightarrow$ & $\sqrt{2 / 3} d_{-1,0}^{2}$ & $d_{-1,1}^{2}$ & $d_{-1,-1}^{2}$ & $2 d_{-1,2}^{2}$ & $2 d_{-1,-2}^{2}$ \\
\hline$V_{+} V_{-} \rightarrow$ & $2 \sqrt{2 / 3} d_{2,0}^{2}$ & $2 d_{2,1}^{2}$ & $2 d_{2,-1}^{2}$ & $4 d_{2,2}^{2}$ & $4 d_{2,-2}^{2}$ \\
\hline$V_{-} V_{+} \rightarrow$ & $2 \sqrt{2 / 3} d_{-2,0}^{2}$ & $2 d_{-2,1}^{2}$ & $2 d_{-2,-1}^{2}$ & $4 d_{-2,2}^{2}$ & $4 d_{-2,-2}^{2}$ \\
\hline \hline
\end{tabular}

TABLE I: Scattering amplitudes for scalars, fermions, and vector bosons via $s$-channel graviton exchange in terms of the Wigner $d$ functions. The subscripts on the particles indicate their helicities. All particle masses have been neglected. An overall factor $-2 \pi G_{N} E_{C M}^{2}$ has been extracted from the amplitudes.

We calculate the scattering amplitude, in the high-energy limit, of all $s$-channel processes with initial- and final-state scalars, fermions, and vector bosons. This covers all the particles of the standard model, as well as the supersymmetric standard model (excluding gravitons and gravitinos). Only certain amplitudes are allowed, due to helicity conservation in the gravitational interaction of the external particles in the massless limit. The nonzero amplitudes are given in 
Table $^{1}$

We now convert these amplitudes to partial-wave amplitudes, $a_{J}$, defined by

$$
\mathcal{A}=16 \pi \sum_{J}(2 J+1) a_{J} d_{\mu, \mu^{\prime}}^{J}
$$

Thus the matrix of $J=2$ partial-wave amplitudes is proportional to the matrix of Table I but with the Wigner $d$ functions discarded, and the term proportional to $d_{0,0}^{0}$ eliminated from the $s \bar{s} \rightarrow s^{\prime} \bar{s}^{\prime}$ amplitude.

We wish to diagonalize the matrix of partial-wave amplitudes when there are $N_{s}$ scalars, $N_{f}$ fermions, and $N_{V}$ vector bosons. To simplify the task, let us combine the states $f_{+} \bar{f}_{-}$and $f_{-} \bar{f}_{+}$into the single state $f_{-} \bar{f}_{+}$, where $f_{-}$ denotes any of the fifteen left-handed states of one generation $\left(u_{R}, u_{G}, u_{B}, \bar{u}_{R}, \bar{u}_{G}, \bar{u}_{B}, d_{R}, d_{G}, d_{B}, \bar{d}_{R}, \bar{d}_{G}, \bar{d}_{B}, e^{-}, e^{+}, \nu\right){ }^{2}$ Since all fermions have the same gravitational interactions (in the massless limit), we should consider the normalized state obtained by summing over all fermion-antifermion states, $\left(1 / \sqrt{N_{f}}\right) \Sigma f_{-} \bar{f}_{+}$. The same is true of the scalars and the vector bosons. Furthermore, the states $V_{+} V_{-}$and $V_{-} V_{+}$are identical in the partial-wave amplitudes, so we need only consider the former.

We are thus led to construct the matrix of partial-wave amplitudes in the basis of states $\left(1 / \sqrt{N_{s}}\right) \Sigma s \bar{s}$, $\left(1 / \sqrt{N_{f}}\right) \Sigma f_{-} \bar{f}_{+}$, and $\left(1 / \sqrt{N_{V}}\right) \Sigma V_{+} V_{-}$, as shown in Table II Each element in the matrix corresponds to a sum of many identical amplitudes. For example, the element in the first row, second column, sums the amplitudes for $N_{s}$ pairs of scalars scattering into $N_{f}$ pairs of fermions. Hence the element is proportional to $N_{s} N_{f}$ times the normalization factors $1 / \sqrt{N_{s}}$ and $1 / \sqrt{N_{f}}$ from the basis states. ${ }^{3}$

\begin{tabular}{|c|c|c|c|}
\hline & $\frac{1}{\sqrt{N_{s}}} \Sigma s^{\prime} \bar{s}^{\prime}$ & $\frac{1}{\sqrt{N}_{f}} \Sigma f_{-}^{\prime} \bar{f}_{+}^{\prime}$ & $\frac{1}{\sqrt{N_{V}}} \Sigma V_{+}^{\prime} V_{-}^{\prime}$ \\
\hline$\frac{1}{\sqrt{N}_{s}} \Sigma s \bar{s} \rightarrow$ & $2 / 3 N_{s}$ & $\sqrt{2 / 3} \sqrt{N_{s} N_{f}}$ & $2 \sqrt{2 / 3} \sqrt{N_{s} N_{V}}$ \\
\hline$\frac{1}{\sqrt{N}_{f}} \Sigma f_{-} \bar{f}_{+} \rightarrow$ & $\sqrt{2 / 3} \sqrt{N_{s} N_{f}}$ & $N_{f}$ & $2 \sqrt{N_{f} N_{V}}$ \\
\hline$\frac{1}{{\frac{1}{N_{V}}}} \Sigma V_{+} V_{-} \rightarrow$ & $2 \sqrt{2 / 3} \sqrt{N_{s} N_{V}}$ & $2 \sqrt{N_{f} N_{V}}$ & $4 N_{V}$ \\
\hline \hline
\end{tabular}

TABLE II: $J=2$ partial-wave amplitudes for $N_{s}$ scalars, $N_{f}$ fermions, and $N_{V}$ vector bosons via $s$-channel graviton exchange. The subscripts on the particles indicate their helicities. An overall factor $-G_{N} E_{C M}^{2} / 40$ has been extracted from the partial-wave amplitudes.

Unitarity demands that the eigenvalues of the matrix of partial-wave amplitudes satisfy

$$
\operatorname{Im} a_{J} \geq\left|a_{J}\right|^{2}
$$

which implies that $\left|\operatorname{Re} a_{J}\right| \leq 1 / 2$. The matrix in Table has only one nonzero eigenvalue,

$$
a_{2}^{(1)}=-\frac{1}{40} G_{N} E_{C M}^{2}\left(\frac{2}{3} N_{s}+N_{f}+4 N_{V}\right),
$$

corresponding to the (unnormalized) eigenvector $\sqrt{\frac{2}{3}} \Sigma s \bar{s}+\Sigma f_{-} \bar{f}_{+}+2 \Sigma V_{+} V_{-}$. Tree unitarity is therefore violated at an energy squared of

$$
E_{C M}^{2}=20\left(G_{N} N\right)^{-1}, \quad N \equiv \frac{2}{3} N_{s}+N_{f}+4 N_{V} .
$$

In the standard model with one Higgs doublet and three generations of fermions, $N_{s}=2, N_{f}=45$, and $N_{V}=12$. Thus tree unitarity is violated at an energy $E_{C M}=\sqrt{60 / 283} G_{N}^{-1 / 2} \approx 6 \times 10^{18} \mathrm{GeV}$. This is about one half of the Planck mass.

\footnotetext{
1 These amplitudes are calculated using the Feynman rules of Ref. [13], the spinors of Ref. 14], and the polarization vectors of Ref. [15]. The Wigner $d$ functions may be found in Ref. [16].

2 We are assuming that neutrinos are Majorana fermions, and that the left-handed state $\bar{\nu}$ is not present in the theory.

3 The diagonal elements of this matrix include processes in which the initial and final states are identical. We are ignoring the $t$ - and $u$-channel contributions to these amplitudes, for reasons discussed above. The $s$-channel contribution by itself is gauge invariant.
} 


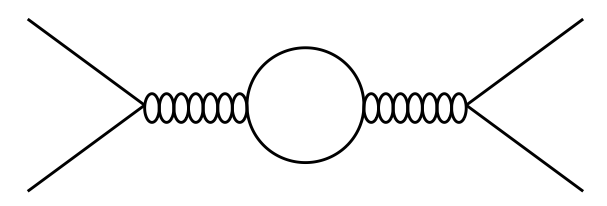

FIG. 2: One-loop correction to the scattering of elementary particles via $s$-channel graviton exchange. The loop contains a sum over all elementary particles.

If nature is supersymmetric, then there are many more particles to consider, since every standard-model particle has a superpartner. ${ }^{4}$ In the minimal supersymmetric standard model with two Higgs doublets, $N_{s}=45+4=49$, $N_{V}=12$, and $N_{f}=N_{s}+N_{V}=61$. Unitarity is violated at an energy $E_{C M}=\sqrt{12 / 85} G_{N}^{-1 / 2} \approx 4 \times 10^{18} \mathrm{GeV}$. This is about one third of the Planck mass.

Alternatively, one could consider the $J=0$ partial-wave amplitude, which only receives a contribution from scalars. One finds that tree unitarity is violated at an energy squared of

$$
E_{C M}^{2}=6\left(G_{N} N_{s}\right)^{-1} /(1+12 a)^{2}
$$

We do not know the value of $a$, so let us choose $a=0$ for illustrative purposes. With this choice, tree unitarity is violated in the standard model at $E_{C M}=\sqrt{3} G_{N}^{-1 / 2}$, while in the minimal supersymmetric standard model it is violated at $E_{C M}=\sqrt{6} / 7 G_{N}^{-1 / 2} \approx 4 \times 10^{18} \mathrm{GeV}$.

It is easy to understand the origin of the factor $N \equiv \frac{2}{3} N_{s}+N_{f}+4 N_{V}$ that appears in the partial-wave amplitude, Eq. (5). Consider the $s$-channel elastic scattering of the state given by the eigenvector $\sqrt{\frac{2}{3}} \Sigma s \bar{s}+\Sigma f_{-} \bar{f}_{+}+2 \Sigma V_{+} V_{-}$, represented by the tree diagram in Fig. 1 The one-loop correction to the amplitude due to particles other than gravitons is given by the diagram in Fig. 2] where the loop contains scalars, fermions, and vector bosons. Elastic unitarity, via Eq. (4) (with the inequality saturated), tells us that the imaginary part of the $J=2$ partial wave of this one-loop diagram equals the square of the $J=2$ partial wave of the tree diagram. Thus the factor $N$ is simply the weighted sum over all the particles that circulate in the loop.

The $J=2$ partial-wave amplitude to one-loop order is

$$
a_{2}=a_{2}^{(1)}\left(1+\operatorname{Re} a_{2}^{(2)} / a_{2}^{(1)}+i a_{2}^{(1)}\right)
$$

where the superscript in parentheses indicates the order in $G_{N} E^{2}$. We have used $\operatorname{Im} a_{2}^{(2)}=\left|a_{2}^{(1)}\right|^{2}$ in the above equation, as well as the fact that $a_{2}^{(1)}$, given by Eq. (5), is real. The unitarity condition $\left|\operatorname{Re} a_{2}\right| \leq 1 / 2$ implies $\left|a_{2}^{(1)}\right| \leq 1 / 2$ at tree level. We see that this corresponds to the imaginary part of the one-loop correction being less than half of the tree-level amplitude. This is a reasonable criterion for the convergence of the perturbative expansion.

The real part of the one-loop correction, $\operatorname{Re} a_{2}^{(2)}$, is ultraviolet divergent. This divergence is absorbed into the coefficients of the $R^{2}$ and $R^{\mu \nu} R_{\mu \nu}$ terms in the Lagrangian [2]. Thus the coefficients of these terms, relative to the coefficient of the $R$ term in the Lagrangian of Eq. (1), are of order $G_{N} N$, rather than simply $G_{N}$.

Since $N$ is large, it is interesting to consider the large- $N$ limit of the scattering amplitude, defined as $N \rightarrow \infty$ while keeping $G_{N} N$ fixed. In this limit, the leading correction to the scattering amplitude at $n^{\text {th }}$ order arises from the $(n-1)^{\text {th }}$ iteration of the one-loop diagram, as shown in Fig. 3 [17. One can sum the geometric series generated by these leading corrections, to obtain

$$
a_{2}=\frac{a_{2}^{(1)}}{1-\operatorname{Re} a_{2}^{(2)} / a_{2}^{(1)}-i a_{2}^{(1)}} .
$$

This expression for the $J=2$ partial wave satisfies elastic unitarity, Eq. (4) (with the inequality saturated), exactly, regardless of the size of $a_{2}^{(1)}$. Thus, at leading order in $1 / N$, unitarity is respected at arbitrary energies, despite the violation of tree unitarity at the energy given in Eq. (6).

\footnotetext{
4 The superpartners of the vector bosons are Majorana fermions, so $\bar{f}=f$ in the scattering amplitudes. The same is true of Majorana neutrinos.
} 


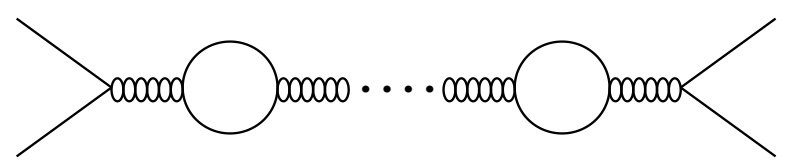

FIG. 3: Multi-loop correction to the scattering of elementary particles via $s$-channel graviton exchange in the large- $N$ limit. Each loop contains a sum over all elementary particles.

The amplitude in Eq. (9) holds on the positive, real $s$ axis above the branch cut. More generally, the amplitude in the complex $s$ plane, in the large- $N$ limit, may be obtained from Eq. (9) by analytic continuation:

$$
a_{2}=\frac{a_{2}^{(1)}}{1+\frac{1}{\pi} a_{2}^{(1)} \ln \left(-\frac{s}{\mu^{2}}\right)},
$$

where $\mu^{2}$ is an arbitrary renormalization scale. This amplitude is unitary, but a pair of complex-conjugate poles on the physical sheet violate the usual analyticity properties. These poles yield acausal effects which become appreciable at energies near $\left(G_{N} N\right)^{-1 / 2}[17$.

In this paper we have found that tree unitarity is violated in the effective field theory of quantum gravity coupled to $N_{s}$ scalars, $N_{f}$ fermions, and $N_{V}$ vectors, at an energy squared of $E_{C M}^{2}=20\left(G_{N} N\right)^{-1}$, where $N=\frac{2}{3} N_{s}+N_{f}+4 N_{V}$. We showed that this is related to radiative corrections, proportional to $G_{N} N E^{2}$, due to loops of such particles in the graviton propagator. New physics must enter before $E_{C M} \approx 6 \times 10^{18} \mathrm{GeV}$ in the standard model, and $4.6 \times 10^{18} \mathrm{GeV}$ in the minimal supersymmetric standard model.

\section{Acknowledgments}

We are grateful for conversations with D. Chung, C. Goebel, J. Harvey, A. Manohar, J. Polchinski, J. Rosner, I. Rothstein, G. Valencia, M. Voloshin, L. Wang, T. Weiler, and E. Weinberg. We thank the Aspen Center for Physics for hospitality. This work was supported in part by the U. S. Department of Energy under contracts Nos. DEFG02-95ER40896 and DE-FG02-91ER40677, by the Wisconsin Alumni Research Foundation, by the National Science Foundation under Grant No. PHY99-07949, and by National Natural Science Foundation of China.

[1] S. Weinberg, Physica A 96, 327 (1979).

[2] J. F. Donoghue, Phys. Rev. D 50, 3874 (1994) arXiv:gr-qc/9405057.

[3] C. P. Burgess, arXiv:gr-qc/0311082

[4] J. Polchinski, String theory, Vol. 2: Superstring theory and beyond, p.

[5] T. D. Lee and C. N. Yang, Phys. Rev. Lett. 4, 307 (1960).

[6] M. S. Chanowitz and M. K. Gaillard, Nucl. Phys. B 261, 379 (1985).

[7] X. G. He, Phys. Rev. D 61, 036007 (2000) arXiv:hep-ph/9905500; T. Han, G. D. Kribs and B. McElrath, Phys. Rev. D 64, 076003 (2001) arXiv:hep-ph/0104074; R. S. Chivukula, D. A. Dicus, H. J. He and S. Nandi, Phys. Lett. B 562, 109 (2003) arXiv:hep-ph/0302263; S. De Curtis, D. Dominici and J. R. Pelaez, Phys. Rev. D 67, 076010 (2003) arXiv:hep-ph/0301059.

[8] C. G. Callan, S. R. Coleman and R. Jackiw, Annals Phys. 59, 42 (1970).

[9] M. J. Veltman, in Methods in Field Theory, Proceedings of the 1975 Les Houches Summer School, eds. R. Bailin and J. Zinn-Justin (North Holland, Amsterdam, 1976), p. 265.

[10] G. 't Hooft and M. J. Veltman, Annales Poincare Phys. Theor. A 20, 69 (1974).

[11] R. P. Feynman, F. B. Morinigo, W. G. Wagner and B. Hatfield, Feynman Lectures On Gravitation (Addison-Wesley, Reading, 1995).

[12] A. Datta, E. Gabrielli and B. Mele, Phys. Lett. B 579, 189 (2004) arXiv:hep-ph/0309232.

[13] T. Han, J. D. Lykken and R. J. Zhang, Phys. Rev. D 59, 105006 (1999) arXiv:hep-ph/9811350.

[14] S. Willenbrock, in Techniques and Concepts in High-Energy Physics XII, Proceedings of the NATO Advanced Study Institute, St. Croix, U. S. Virgin Islands, 2002, eds. H. Prosper and M. Danilov (Kluwer, Dordrecht, 2003), p. 1 arXiv:hep-ph/0211067.

[15] K. Hagiwara and D. Zeppenfeld, Nucl. Phys. B 274, 1 (1986).

[16] K. Hagiwara et al. [Particle Data Group Collaboration], Phys. Rev. D 66, 010001 (2002). 
[17] E. Tomboulis, Phys. Lett. B 70, 361 (1977); E. Tomboulis, Phys. Lett. B 97, 77 (1980). 